\title{
Topic 10: Parallel Numerical Algorithms
}

\author{
Michel Cosnard, Hans-Joachim Bungartz, \\ Efstratios Gallopoulos, and Yousef Saad
}

Topic Chairs

Since the early days of supercomputing, numerical routines have caused the highest demand for computing power anywhere, making their efficient parallelization one of the core methodical tasks in high-performance computing. And still, many of today's fastest computers in the world are mostly used for the solution of huge systems of equations as they arise in the simulation of complex large scale problems in engineering and science.

Despite this long tradition, parallel numerical algorithms did not lose anything of their relevance. The efficient implementation of existing schemes on stateof-the-art parallel systems (such as clusters or hybrid systems), the challenges resulting from massively parallel systems, the design of easy-to-use portable software components, the recent endeavours to tackle optimization, control, and interactive steering scenarios, too - all this clearly shows that progress in computational science and engineering strongly depends on progress with parallel numerical algorithms. This crucial importance of parallel numerical algorithms certainly justifies to again having devoted a special workshop to this topic at Euro-Par, in addition to the discussion of special aspects of high-performance and grid computing in Topic 16.

Overall, fourteen papers were submitted to our Topic. With authors from Denmark, France, Germany, Greece, Lithuania, Spain, and Switzerland, Europe is the dominant male (as expected at Euro-Par), but three papers are authored by scientists from the United States, Australia, and China. Out of these fourteen submissions, eight were accepted as regular papers.

Both devising new parallel algorithms for numerical tasks and adapting existing ones to state-oft-the-art parallel systems are vigorously flourishing and still developing fields of research. Hence, it is no surprise that the eight research articles presented in this section cover a wide range of topics arising in the various subdomains of parallel numerical algorithms. At the conference, the presentations were arranged into three sessions on PDE-Related Topics, ODE- or Particle-Related Topics, and Miscellaneous Topics. This structure also reflects in the following part of the conference's proceedings.

In the PDE-Related Topics section, Raimondas Ciegis addresses a parallel locally one-dimensional scheme for the numerical solution of three-dimensional parabolic problems with nonlocal boundary conditions. Vincent Heuveline and Andrea Walther discuss online checkpointing strategies for parallel adjoint computations as they occur in the optimization or control of time-dependent flow problems. Finally, fault tolerance is the topic of Hatem Ltaief, Marc Garbey, and Edgar Gabriel. In their contribution, they study parallel fault tolerant algorithms for parabolic problems. 
Ordinary differential equations and particle methods are the central topics of the second section. José M. Badía, Peter Benner, Rafael Mayo, and Enrique Quintana-Ortí deal with generalized algebraic Riccati equations, especially parallel schemes for large-scale and sparse ones. Load balancing strategies and their applicability to data-parallel embedded Runge-Kutta integrators are studied by Matthias Korch and Thomas Rauber. The third presentation, given by a team of eight scientists from ETH Zürich, focuses on a software framework for the portable parallelization of particle-mesh simulations, the combination of particleand mesh-based methods.

The final third session gathers the two remaining papers of Topic 10. Rita Zrour, Pierre Chatelier, Fabien Feschet, and Rémy Malgouyres study the parallelization of a numerical and computationally intense problem from computer graphics: discrete radiosity methods for global illumination. Finally, an interdisciplinary team of five researchers from chemistry and computer science at TU München addresses the parallelization of matrix operations as a typical building block from numerical linear algebra, as they appear in optimal control-based quantum compilers.

Altogether, the contributions to Topic 10 at the 2006 Euro-Par in Dresden show once more the great variety of interesting, challenging, and important issues in the field of parallel numerical algorithms. Thus, we are already looking forward to the new results submitted to and presented at next year's Euro-Par conference. 